\title{
Muscle regeneration after sepsis
}

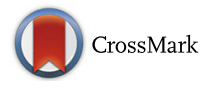

\author{
Adrien Bouglé (1, $^{*}$, Pierre Rocheteau', Tarek Sharshar ${ }^{1,3,4,5}$ and Fabrice Chrétien ${ }^{1,5,6,7}$
}

\begin{abstract}
Severe critical illness is often complicated by intensive care unit-acquired weakness (ICU-AW), which is associated with increased ICU and post-ICU mortality, delayed weaning from mechanical ventilation and longterm functional disability. Several mechanisms have been implicated in the pathophysiology of ICU-AW, but muscle regeneration has not been investigated to any extent in this context, even though its involvement is suggested by the protracted functional consequences of ICU-AW. Recent data suggest that muscle regeneration could be impaired after sepsis, and that mesenchymal stem cell treatment could improve the post-injury muscle recovery.
\end{abstract}

The primary functions of skeletal musculature are locomotor activity, postural behavior, and breathing. Severe critical illness is often complicated by intensive care unitacquired weakness (ICU-AW), which is clinically characterized by bilateral and symmetrical limb weakness and is related to a myopathy and/or axonal polyneuropathy. ICU-AW affects between $25 \%$ and $60 \%$ of patients mechanically ventilated for more than 7 days [1], and is associated with increased ICU and post-ICU mortality, delayed weaning from mechanical ventilation and long-term functional disability [2]. Most patients who develop ICU-AW have been admitted for a sepsis episode, and the main risk factors for ICU-AW include the severity of critical illness, immobilization, hyperglycemia, and the use of some medications, including steroids and neuromuscular agents, although this is somewhat controversial.

The pathophysiology of critical illness myopathy is thought to involve the following mechanisms: 1) impairment of muscular membrane excitability, secondary to a dysregulation of sodium channel gating [3];

\footnotetext{
* Correspondence: adrien.bougle@aphp.fr

${ }^{1}$ Human Histopathology and Animal Models Unit, Infection and Epidemiology Department, Institut Pasteur, 75724 cedex15 Paris, France

${ }^{2}$ Anesthesiology and Intensive Care, Institut de Cardiologie, Hôpitaux

Universitaires La Pitié-Salpêtrière, Paris 75013, France

Full list of author information is available at the end of the article
}

2) mitochondrial dysfunction leading to bioenergetic failure and oxidative stress [4]; and 3) proteolysis, mainly related to an activation of the ubiquitin-proteasome pathway [5]. These mechanisms can be triggered by various factors, notably systemic inflammatory mediators, endocrine dysfunction, immobilization, some drugs, and electrolyte disturbances. The protracted functional consequences of ICU-AW indicate that muscle regeneration is also impaired. Surprisingly, muscle regeneration, which essentially depends on the muscle stem cells (also called satellite cells (SC)), has not been extensively investigated in the context of critical illness. SC that are located at the periphery of the muscle fiber [6] are activated in response to any muscle injury and then proliferate and differentiate to repair or replace the damaged fibers, and also self-renew to replenish the muscle stem cell reservoir [7].

It was recently demonstrated in a murine model of polymicrobial peritonitis that SC activation, proliferation, and expression of myogenic markers were impaired after sepsis, leading to impaired muscle regeneration; however, the post-sepsis intramuscular administration of exogenous mesenchymal stem cells (MSCs) could reverse this SC dysfunction [8]. MSC treatment significantly improved the post-injury muscle recovery with decreasing necrosis and fibrosis but also increased the force of isolated single fibers. It is conceivable that a systemic anti-inflammatory effect of MSCs is involved, as their administration induced a decrease in the plasma levels of pro-inflammatory cytokines and procalcitonin. MSCs were previously shown to possess immunomodulatory effects via interaction with immune cells [9-11], the MSC secretome [12], and transfer of mitochondrial material [13]. Furthermore, MSC treatment enhances bacterial clearance during infections [14]. These different capabilities led researchers to test this treatment in two severe conditions frequently encountered in the ICU: acute respiratory distress syndrome (ARDS) and sepsis. A recently published review on 54 pre-clinical studies reported that treatment with MSCs could significantly decrease mortality in animals with acute respiratory failure [15]. MSC treatment has been tested in phase 1 trials [16], and clinical trials are on the way. Likewise, experimental 
studies in sepsis showed that MSC treatment after polymicrobial sepsis could reduce mortality and improve organ function [17], but also prevent the occurrence of muscle weakness or accelerate muscle recovery. One may argue that, beforehand, we need to: 1) make sure that critical illness myopathy is associated with SC dysfunction in patients; 2) understand why the sepsis has such a sustainable impact on SC which have been shown to resist anoxia up to 17 days after death [18]; and 3) investigate comprehensively the interactions between SC and MSCs, notably in the context of sepsis. The route and time of administration in patients must be addressed. Only a stepwise and comprehensive approach would allow us to determine whether or not MSCs are truly efficient, and also would enable us to identify new therapeutic targets.

\section{Abbreviations}

ARDS: acute respiratory distress syndrome; ICU-AW: intensive care unitacquired weakness; MSC: mesenchymal stem cell; SC: satellite cells.

\section{Competing interests}

The authors declare that they have no competing interests.

\section{Authors' contributions}

$A B$ drafted the manuscript. PR helped to draft the manuscript. TS and FC helped to revise the manuscript. All authors read and approved the final manuscript.

\section{Author details}

'Human Histopathology and Animal Models Unit, Infection and Epidemiology Department, Institut Pasteur, 75724 cedex15 Paris, France. ${ }^{2}$ Anesthesiology and Intensive Care, Institut de Cardiologie, Hôpitaux Universitaires La Pitié-Salpêtrière, Paris 75013, France. Intensive Care, Hôpital Raymond Poincaré, Garches 92380, France. ${ }^{4}$ Versailles Saint Quentin University, Versailles 78000, France. ${ }^{5}$ TRIGGERSEP, F-CRIN Network, Versailles 78000, France. ${ }^{6}$ Neuropathology Laboratory, Centre Hospitalier Sainte Anne, Paris 75014, France. ${ }^{7}$ Paris Descartes University, Sorbonne Paris Cité, Paris 75006, France.

\section{Published online: 19 May 2016}

\section{References}

1. de Jonghe B, Sharshar T, Lefaucheur J-P, Authier F-J, Durand-Zaleski I, Boussarsar $M$, et al. Paresis acquired in the intensive care unit: a prospective multicenter study. JAMA. 2002;288:2859-67.

2. Sharshar T, Bastuji-Garin S, Stevens RD, Durand M-C, Malissin I, Rodriguez P, et al. Presence and severity of intensive care unit-acquired paresis at time of awakening are associated with increased intensive care unit and hospital mortality. Crit Care Med. 2009;37:3047-53.

3. Teener JW, Rich MM. Dysregulation of sodium channel gating in critical illness myopathy. J Muscle Res Cell Motil. 2006;27:291-6.

4. Brealey D, Brand M, Hargreaves I, Heales S, Land J, Smolenski R, et al. Association between mitochondrial dysfunction and severity and outcome of septic shock. Lancet. 2002;360:219-23.

5. Ochala J, Gustafson A-M, Diez ML, Renaud G, Li M, Aare S, et al. Preferential skeletal muscle myosin loss in response to mechanical silencing in a novel rat intensive care unit model: underlying mechanisms. J Physiol (Lond). 2011:589:2007-26

6. Lepper C, Partridge TA, Fan C-M. An absolute requirement for Pax7-positive satellite cells in acute injury-induced skeletal muscle regeneration. Development. 2011;138:3639-46.

7. Gayraud-Morel B, Chrétien F, Tajbakhsh S. Skeletal muscle as a paradigm for regenerative biology and medicine. Regen Med. 2009;4:293-319.

8. Rocheteau P, Chatre L, Briand D, Mebarki M, Jouvion G, Bardon J, et al. Sepsis induces long-term metabolic and mitochondrial muscle stem cell dysfunction amenable by mesenchymal stem cell therapy. Nat Commun. 2015;6:10145.
9. Krampera M, Glennie S, Dyson J, Scott D, Laylor R, Simpson E, et al. Bone marrow mesenchymal stem cells inhibit the response of naive and memory antigen-specific T cells to their cognate peptide. Blood. 2003:101:3722-9.

10. Corcione A, Benvenuto F, Ferretti E, Giunti D, Cappiello V, Cazzanti F, et al. Human mesenchymal stem cells modulate B-cell functions. Blood. 2006;107:367-72.

11. Rafei M, Hsieh J, Fortier S, Li M, Yuan S, Birman E, et al. Mesenchymal stromal cell-derived CCL2 suppresses plasma cell immunoglobulin production via STAT3 inactivation and PAX5 induction. Blood. 2008;112:4991-8.

12. Tse WT, Pendleton JD, Beyer WM, Egalka MC, Guinan EC. Suppression of allogeneic T-cell proliferation by human marrow stromal cells: implications in transplantation. Transplantation. 2003;75:389-97.

13. Islam MN, Das SR, Emin MT, Wei M, Sun L, Westphalen $K$, et al. Mitochondrial transfer from bone-marrow-derived stromal cells to pulmonary alveoli protects against acute lung injury. Nat Med. 2012;18:759-65.

14. Mei SHJ, Haitsma JJ, dos Santos CC, Deng Y, Lai PFH, Slutsky AS, et al. Mesenchymal stem cells reduce inflammation while enhancing bacterial clearance and improving survival in sepsis. Am J Respir Crit Care Med. 2010;182:1047-57.

15. McIntyre LA, Moher D, Fergusson DA, Sullivan KJ, Mei SHJ, Lalu M, et al. Efficacy of mesenchymal stromal cell therapy for acute lung injury in preclinical animal models: a systematic review. PLoS One. 2016;11, e0147170.

16. Wilson JG, Liu KD, Zhuo H, Caballero L, McMillan M, Fang X, et al. Mesenchymal stem (stromal) cells for treatment of ARDS: a phase 1 clinical trial. Lancet Respir Med. 2015;3:24-32.

17. Németh K, Leelahavanichkul A, Yuen PST, Mayer B, Parmelee A, Doi K, et al. Bone marrow stromal cells attenuate sepsis via prostaglandin E(2)-dependent reprogramming of host macrophages to increase their interleukin-10 production. Nat Med. 2009;15:42-9.

18. Latil M, Rocheteau P, Châtre L, Sanulli S, Mémet S, Ricchetti M, et al. Skeletal muscle stem cells adopt a dormant cell state post mortem and retain regenerative capacity. Nat Commun. 2012;3:903. 